\title{
ANATOMia foliar del gÉNERo Smallanthus (Asteraceae, MiLlerieAe)
}

\author{
MAIRA S. VITALI ${ }^{1}$
}

\begin{abstract}
Summary: Leaf anatomy of the genus Smallanthus (Asteraceae, Millerieae). Given the great medicinal and nutritional importance of the leaf in Smallanthus species, such as the world famous "yacón" (Smallanthus sonchifolius), and that so far only have been studied four of the 23 species that constitute the genus, the leaf anatomy of all species of Smallanthus is analyzed here. A table of uses and vernacular names of the species of Smallanthus and a general description of leaf anatomy, including the petiole is presented. Three types of trichomes were identified: an eglandular simple conical hair, a glandular biseriate vesicular hair, and a glandular biseriate capitate subtype beta hair. Smallanthus species show almost no differences in the anatomy of the blade and petiole. The schizogenous secretory channels show a tendency to increase in number in the southernmost species.
\end{abstract}

Key words: Asteraceae, leaf, trichomes, secretory canals, uses.

Resumen: Debido a la gran importancia medicinal y alimentaria que tienen las hojas en especies de Smallanthus, como por ejemplo el mundialmente conocido "yacón" (Smallanthus sonchifolius), y que hasta el momento solo se han estudiado cuatro especies de las 23 que componen el género, se analizó aquí la anatomía foliar de todas las especies de Smallanthus. Se presenta también, una tabla de usos y nombres vulgares de las especies de las que se obtuvo tal información. Se realiza una descripción general de la anatomía foliar, incluyendo el pecíolo. Se identificaron tres tipos de tricomas: uno eglandular del tipo cónico simple y dos glandulares (biseriado capitado y biseriado vesiculado subtipo beta). Las especies de Smallanthus no mostraron diferencias en la anatomía de la lámina y del pecíolo. Se contabilizaron los canales secretores, de tipo esquizógeno, en casi todas las especies y se observó una tendencia al aumento del número de canales en las especies más australes.

Palabras clave: Asteraceae, hoja, tricomas, canales secretores, usos.

\section{INTRODUCCION}

El género Smallanthus Mackenzie comprende 23 especies de hierbas, arbustos o árboles con tallos huecos y raíces tuberosas, que se distribuyen desde el sudeste de los Estados Unidos hasta el centro-este de Argentina (Vitali et al., 2015). Entre sus especies destaca el "yacón", Smallanthus sonchifolius (Poepp.) H. Rob., originario de la región andina del Perú, que almacena en sus raíces fructosa, glucosa,

1 División Plantas Vasculares, Museo de La Plata, Facultad de Ciencias Naturales y Museo. UNLP. E-mail: vitali@fcnym.unlp.edu.ar sacarosa, oligosacáridos, residuos de almidón e inulina. (Asami et al., 1989; Ohyama et al., 1990). Posee también, asociadas principalmente a las hojas, importantes propiedades medicinales y/o alimentarias tales como, efecto hipoglucemiante (Aybar et al., 2001), antitumorales (De Ford et al., 2015), para problemas renales, como prebiótico (Lachman et al., 2003) y control de la obesidad (Mendoza Meza, et al., 2015). Choque Delgado et al. (2013) consideran al "yacón" como un alimento funcional que, según Valencia \& Román (2004), es todo aquel que, además de sus funciones nutricionales básicas, es capaz de producir efectos metabólicos o fisiológicos útiles en el mantenimiento de una buena salud física y mental. Otras especies que se han estudiado dentro del género también 
poseen importantes propiedades medicinales tales como Smallanthus connatus (Spreng.) H. Rob. (Bach et al., 2007) y S. macroscyphus (Baker) A. Grau ex Sáenz in Zuloaga \& Morrone (Cabrera et al., 2007). Por otro lado, el análisis de cerca de 600 ejemplares durante la realización de la revisión sistemática de Smallanthus (Vitali, 2013) reveló usos en otras especies (Tabla 1). La tabla 1 brinda un panorama orientador, para futuros estudios, sobre aquellas especies de Smallanthus con potenciales propiedades/usos. Desde el punto de vista de la morfología foliar, este género posee una gran variación tanto de la forma, como del tamaño de las hojas (Vitali et al., 2015). Cuatro de las 23 especies fueron analizadas con gran detalle en su anatomía foliar: S. sonchifolius (Mercado et al., 2006), S. macroscyphus, (Coll Aráoz et al., 2008), S. siegesbeckius (DC.) H. Rob. (Mercado et al., 2009) y S. connatus (Coll Aráoz et al., 2012). En estos trabajos no se encontró variación anatómica, ni caracteres diagnósticos que permitieran separar estas cuatro especies. Debido a la importancia medicinal y alimentaria que tienen las hojas como órgano portador de sustancias químicas, se pone de manifiesto la necesidad de realizar un análisis de su anatomía en todas las especies de este género, la mayoría de las cuales hasta el momento no han sido analizadas. Este estudio también incluye los tricomas foliares de los cuales se han mencionado sustancias con actividad antimicrobiana (Inoue et al., 1995) y antidiabética (Genta et al., 2010) y que son responsables de que el follaje sea poco afectado por insectos y pestes (Grau \& Rea, 1997 y Seminario et al., 2003).

\section{Materiales y Metodos}

Los materiales estudiados proceden de los siguientes herbarios nacionales e internacionales: F, G, GH, ICN, K, LP, QCNE, S, SL y TEX (Holmgren et al., 1990, Apéndice). Se analizaron todas las especies del género excepto S. apus (S. F. Blake) H. Rob., ya que solo se contaba con material fotográfico. Para $S$. connatus se contó además con material fresco. En la mayoría de los casos se realizaron cortes transversales de dos y tres ejemplares diferentes de cada especie. En la gran mayoría de las especies, las hojas poseen grandes dimensiones $(6-55 \mathrm{~cm}$ de largo y $2-30 \mathrm{~cm}$ de ancho) por lo que los cortes se realizaron en estos casos a nivel de las venas secundarias en lugar de la vena central principal. Asimismo, los cortes de pecíolo se pudieron realizar en dos especies, $S$. connatus y $S$. macroscyphus, ya que se contó con material fresco y material de herbario posible de cortar sin necesidad de mutilar el ejemplar. El material de herbario se recuperó colocándolo en agua con detergente durante 24 horas. Las muestras se aclararon con hipoclorito de sodio al $50 \%$ y se tiñeron luego con safranina diluida al $2 \%$. Los cortes anatómicos fueron realizados a mano alzada y para la observación de los estomas, se realizó levantamiento de la epidermis y observaciones en superficie. El material fue montado en gelatinaglicerina. Las fotomicrografías fueron realizadas utilizando un microscopio Nikon Eclipse E 200, con cámara digital incorporada. La terminología de tricomas sigue a Ramayya (1962).

\section{Resultados}

En general, la mayor variación se encontró en los tricomas. La anatomía foliar se mantuvo relativamente constante en todas las especies. Por ello, se realizó primero una descripción general de la anatomía de la lámina a nivel genérico, mencionando los casos en que hay alguna variación específica y, a continuación, se describió la anatomía del pecíolo y el indumento foliar.

Lámina: Se observó un estrato epidérmico con células isodiamétricas o rectangulares en sección transversal. Todas las especies analizadas presentaron colénquima angular de hasta 5 capas de células a la altura de la vena media y en posición subepidérmica (Fig. 1A). Los haces vasculares colaterales (Fig. 1B) y el mesófilo dorsiventral con las células del parénquima esponjoso isodiamétricas y con una o dos capas de parénquima en empalizada (Fig. 1C). En el parénquima alrededor de la vena principal, se encontraron canales secretores de tipo esquizógeno en número que varió desde uno en $S$. uvedalius (L.) Mack. (Fig. 2A) hasta 10 en S. connatus (Fig. 2B). También se observaron canales secretores en el parénquima esponjoso del mesófilo (Fig. 2C). Los estomas de tipo anomocíticos, se encontraron elevados o no sobre la superficie (Fig. 3). 
Tabla 1. Resumen de la información obtenida, de etiquetas de herbarios y de la bibliografia, sobre los usos y nombres vulgares de especies de Smallanthus.

\begin{tabular}{|c|c|c|c|}
\hline Especie & Usos & $\begin{array}{l}\text { Fuente de informacion } \\
\text { (bibliografia y/o } \\
\text { etiqueta de herbario }\end{array}$ & Nombre vulgar \\
\hline S. connatus & $\begin{array}{l}\text { Hojas: hipoglucemiante. } \\
\text { Raíces: hipoglucemiante, } \\
\text { analgésica, antitumoral, anti-HIV, } \\
\text { antimicrobiana, antiinflamatoria. } \\
\text { Semillas: combustible. }\end{array}$ & $\begin{array}{l}\text { Coll Aráoz et al., (2012); } \\
\text { Vitali et al., (2015) }\end{array}$ & $\begin{array}{l}\text { Girasolcito, jaguarete, ka'a, } \\
\text { yacón-gaúcho, yacón-nativo. }\end{array}$ \\
\hline S. macroscyphus & $\begin{array}{l}\text { Hipoglucemiante, antitumoral, } \\
\text { antimicrobiano, antifúngico, } \\
\text { antiinflamatorio. }\end{array}$ & $\begin{array}{l}\text { Cabrera et al., (2006); } \\
\text { Ebber (2011) }\end{array}$ & Yacón de campo. \\
\hline S. fruticosus & $\begin{array}{l}\text { Hojas, tallos y flores: anti- } \\
\text { inflamatorios del tracto urinario }\end{array}$ & Cerón 17656 (QCNE) & $\begin{array}{l}\text { Colla, colla culapa, polaco, } \\
\text { shita, shitadeloso. }\end{array}$ \\
\hline & Detergente & & Ax, árnica, balim k'in, \\
\hline S. maculatus & $\begin{array}{l}\text { Hojas: sedante y para el "mal de } \\
\text { ojo", para heridas y ulceras de } \\
\text { las piernas y el tratamiento de } \\
\text { enfermedades gastrointestinales. }\end{array}$ & $\begin{array}{l}\text { Vovides } 26(F) \text {; Balick } \\
\text { et al., } 2216(\mathrm{GH})\end{array}$ & $\begin{array}{l}\text { gua-gua, ik'al, k'ail, } \\
\text { k'ayil, margarita, mirasol, } \\
\text { mirasol de monte, purca, } \\
\text { tora, shti-pú, tz'ibal. }\end{array}$ \\
\hline S. meridensis & Medicinal & Aristeguieta $2484(\mathrm{~F})$ & Escorzonera. \\
\hline S. parviceps & Medicinal y para masticar coca & $\begin{array}{l}\text { Herrera } 1480(\mathrm{GH}) \\
\text { Johns } 81-54(\mathrm{~F})\end{array}$ & $\begin{array}{l}\text { Jareta, shita, wila- } \\
\text { wila, yareta, yarita. }\end{array}$ \\
\hline S. pyramidalis & $\begin{array}{l}\text { Hojas: mitigar el dolor de cabeza. } \\
\text { Para el reumatismo y contra } \\
\text { resfriados y contusiones }\end{array}$ & $\begin{array}{l}\text { Steyermark } 57280(\mathrm{~F}) \text {; } \\
\text { Cerón } 15672(\mathrm{~K}, \mathrm{QCNE}) \text {; } \\
\text { Cerón } 15811 \text { (QCNE) }\end{array}$ & $\begin{array}{l}\text { Anime, anime manso, anime } \\
\text { negro, arbolaco, arboloco, } \\
\text { colla, (f)tajuash, polaco. }\end{array}$ \\
\hline S. siegesbeckia & $\begin{array}{l}\text { Citotóxica, embriotóxica e } \\
\text { inhibidora del crecimiento y } \\
\text { desarrollo larval. Miorrelajante, } \\
\text { analgésica, antitumoral, } \\
\text { antifúngica, antimicrobiana y } \\
\text { anti-HIV. Hojas: antioxidante }\end{array}$ & $\begin{array}{l}\text { Costa Lotufo et al., (2002); } \\
\text { Bresciani et al., (2004); } \\
\text { Ghisalberti (1997) }\end{array}$ & Yarita. \\
\hline S. sonchifolius & $\begin{array}{l}\text { Hipoglucemiante, alto contenido de } \\
\text { potasio, antioxidante, edulcorante. } \\
\text { Fruta seca y fresca (raíces). Té. }\end{array}$ & $\begin{array}{l}\text { Mayta et al., (2004); } \\
\text { Seminario et al., (2003); } \\
\text { Takenaka et al., (2003); } \\
\text { Manrique et al., (2005). }\end{array}$ & $\begin{array}{l}\text { Anime, anime manso, anime } \\
\text { negro, arbolaco, arboloco, } \\
\text { colla, (f)tajuash, polaco } \\
\text { yacón, aricoma, aricuma, } \\
\text { chicama, jicama, jíquima, } \\
\text { jiquimilla, llaqon, llacum, } \\
\text { llacuma, shicama, yacumpi. }\end{array}$ \\
\hline S. uvedalius & $\begin{array}{l}\text { El pueblo Cherokee usaba } \\
\text { las raíces para expulsar la } \\
\text { placenta después del parto. }\end{array}$ & Olbrechts (1931) & Mirasol, paira, purca, xilil. \\
\hline
\end{tabular}

Pecíolo: La morfología del pecíolo es muy variable entre las especies del género, hay pecíolos con alas grandes y anchas, con alas marcadamente atenuadas, y otras especies carecen de pecíolo (Vitali et al., 2015). Anatómicamente sin embargo, el pecíolo resultó ser homogéneo. A la altura de la vena media se observó una cutícula relativamente gruesa sobre una epidermis de una o dos capas de células y por debajo colénquima angular de más de cinco capas de células. Hacia el interior hay un parénquima de células más o menos isodiamétricas, en el cual se encontraron ca. 8 haces vasculares (Fig. 4A), y más de 10 canales secretores (Fig. 4B).

Indumento: Las hojas de todas las especies de Smallanthus presentan algún grado de pubescencia, en una o en ambas caras (Vitali et al., 2015). Los tricomas resultaron ser eglandulares o glandulares encontrándose generalmente ambos tipos (Fig. 


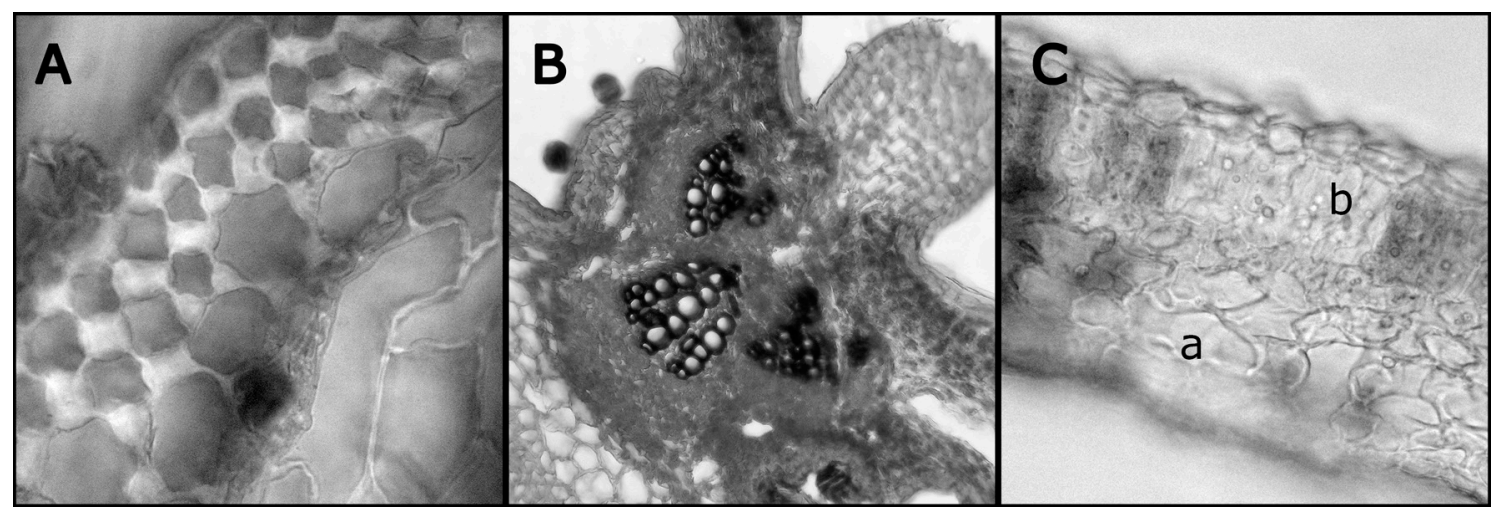

Fig. 1. Anatomía de la lámina en Smallanthus. A. Detalle del colénquima angular subepidérmico en $S$. mcvaughii (Campos 4563). B. Sección transversal de los haces vasculares colaterales en S. glabratus (Macbride 1554). C. Mesófilo dorsiventral de S. lundelli (Contreras 11065) donde se observa en detalle el parénquima esponjoso a. y el parénquima en empalizada $\mathbf{b}$.

5A), excepto en S. glabratus (DC.) H. Rob. que solo presentó escasos tricomas glandulares. Los tipos de tricomas presentes en cada especie se muestran en la Tabla 2.

Tricomas eglandulares: Cónico simple (Fig. 5Aa): presentan un pie simple o compuesto (Fig. 5B), cuerpo uniseriado, de 2 o más células, cónicas, agudas o generalmente redondeadas hacia el ápice. Estos tricomas se encontraron en la superficie adaxial y abaxial de las hojas de la mayoría de las especies y agrupados sobre protuberancias de la superficie foliar a la altura de las venas principales de las hojas (Fig. 5C) de algunas especies (Tabla 2). También fueron observados en estructuras reproductivas, como por ejemplo en la base de las flores del radio formando un ramillete, el cual resultó en la filogenia morfológica de Smallanthus, la sinapomorfía que soporta al género.

Tricomas glandulares: 1) Biseriado capitado: presentan un pie simple o compuesto y un

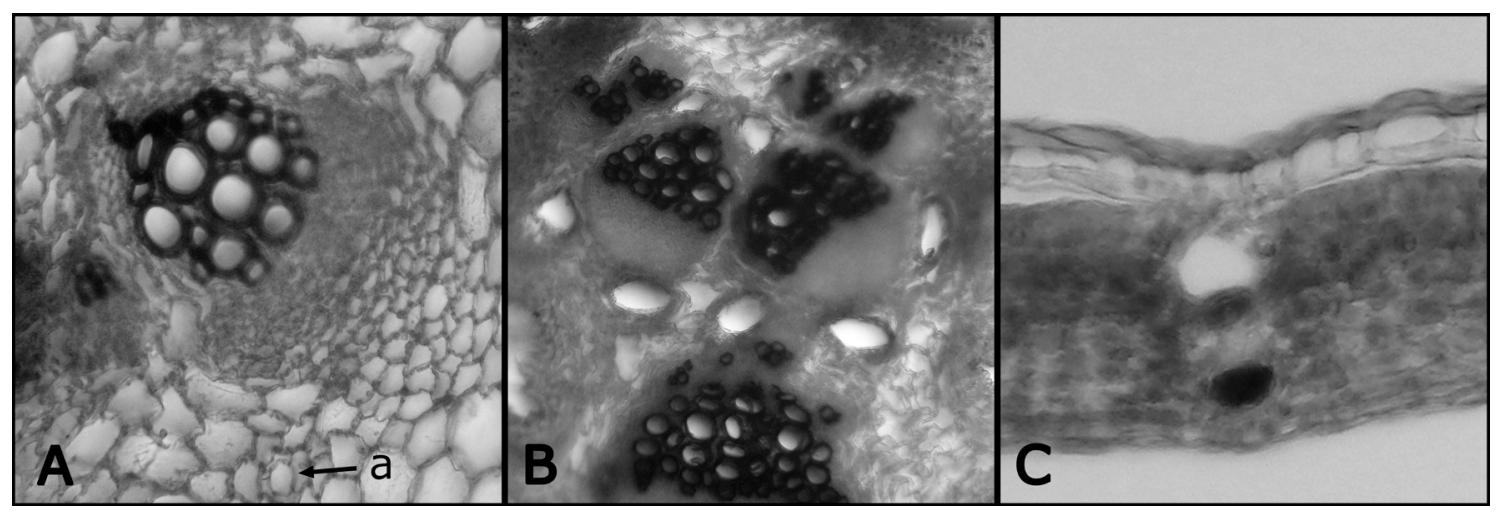

Fig. 2. Canales secretores esquizógenos en el parénquima en torno a la vena principal. A. En sección tranversal, detalle del único canal secretor presente en $S$. uvedalius a. (Kearney $\mathrm{s} / \mathrm{n}$ ). B. Múltiples canales secretores en $S$. connatus (Plos 46). C. Canal secretor en el parénquima del mesófilo de $S$. latisquamus (Chrysler 5544). 


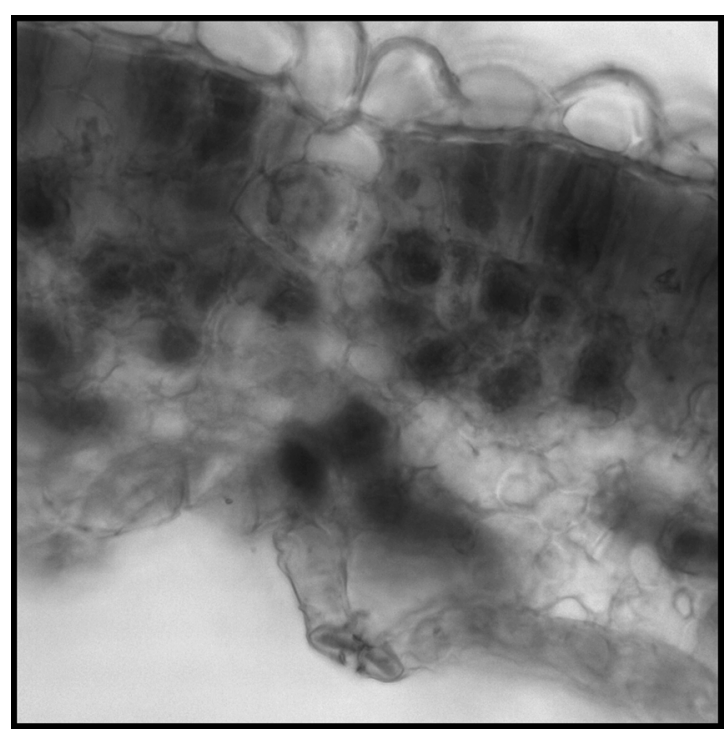

Fig. 3. Estoma en la cara adaxial de la hoja, en sección transversal de $S$. obscurus, que sobresale de la superficie. (Breedlove 42387).

cuerpo biseriado que se diferencia de una cabeza (Fig. 6A). La cabeza está formada por 1 a 5 capas de células de variado tamaño con paredes internas delgadas, siendo las laterales rectas o ligeramente convexas. El cuerpo es de variada longitud, con 3-7 células en cada fila, usualmente estrechándose hacia la cabeza. Estos tricomas se encontraron sobre la superficie adaxial de las hojas de varias especies (Tabla 2) y se observaron también en filarios, en las corolas de las flores del disco, en los tubos de flores del radio y en algunas especies en las páleas y en las lígulas de las flores del radio (Vitali et al., 2015).

2) Biseriado vesiculado subtipo Beta: presenta un pie simple o compuesto (Fig. 6B) y un cuerpo que es biseriado, de 4 a 10 células con forma oblonga o cuneada y las paredes externas levemente convexas (Fig. 6 C). La vesícula cuticular encierra 1-4 células terminales. Estos tricomas se encontraron tanto en la cara adaxial como abaxial de las hojas de algunas especies (Tabla 2) y también se observaron sobre los filarios internos y las lígulas de unas pocas especies (Vitali et al., 2015).

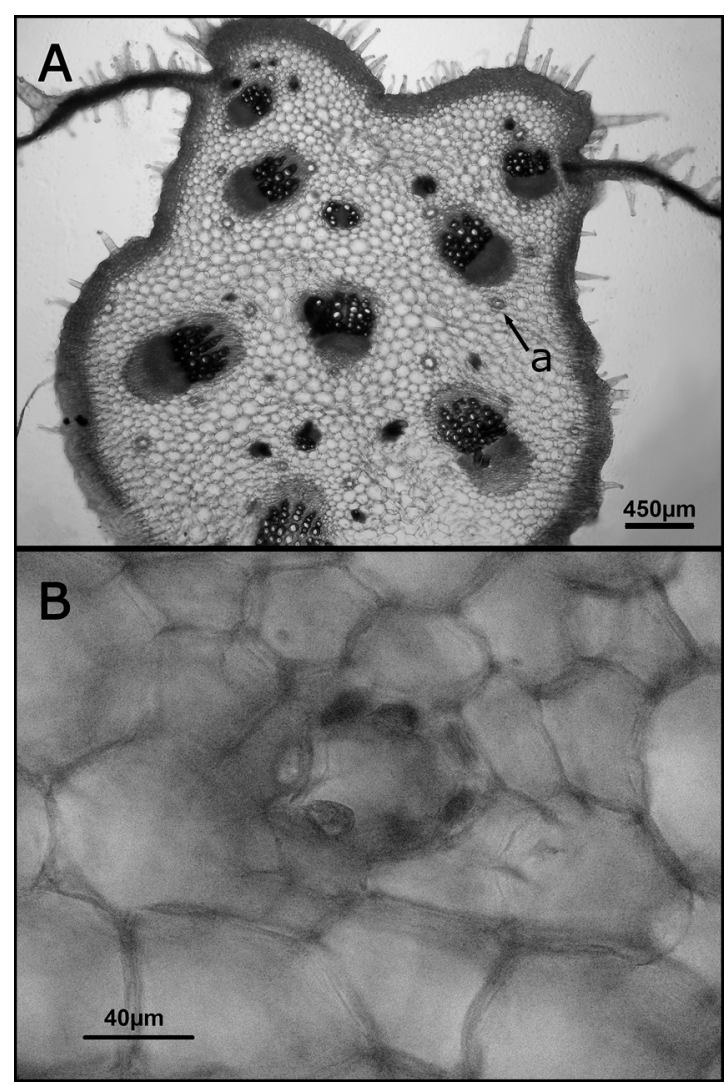

Fig. 4. Anatomía del pecíolo en sección transversal. A. Pecíolo de S. conatus (Plos 46). B. Detalle de uno de los canales secretores del pecíolo.

\section{Discusión}

Esta es la primera vez que se analiza la anatomía foliar de todas las especies de Smallanthus. Este género posee una gran variación de la morfología foliar externa, tanto en la forma como en el tamaño de la lámina (Vitali et al., 2015), y a su vez las especies habitan en ambientes muy disímiles como orillas de ríos (S. connatus), bosques nublados $[S$. latisquamus (S. F. Blake) H. Rob.], y cumbres de ca. 3400 m.s.m. [S. jelskii (Hieron.) H. Rob.]. Por ello, se esperaría encontrar una anatomía foliar que represente dicha variación. Sin embargo, los resultados de este trabajo no arrojan notables diferencias entre las especies. Respecto a los canales secretores, se observaron en todas las especies y se constató que variaban en número 
Bol. Soc. Argent. Bot. 52 (3) 2017

Tabla 2. Tipos de tricomas en especies del género Smallanthus y cuáles de ellas poseen los tricomas agrupados sobre protuberancias a la altura de las venas principales de las hojas.

\begin{tabular}{|c|c|c|c|c|}
\hline Especie & $\begin{array}{l}\text { Tricoma Cónico } \\
\text { simple }\end{array}$ & $\begin{array}{l}\text { Agrupados sobre } \\
\text { protuberancias }\end{array}$ & $\begin{array}{c}\text { Tricoma Biseriado } \\
\text { capitado }\end{array}$ & $\begin{array}{c}\text { Tricoma Biseriado } \\
\text { vesiculado subtipo Beta }\end{array}$ \\
\hline S. connatus & $\mathrm{Si}$ & & $\mathrm{Si}$ & $\mathrm{Si}$ \\
\hline S. cocuyensis & $\mathrm{Si}$ & $\mathrm{Si}$ & & $\mathrm{Si}$ \\
\hline S. fruticosus & $\mathrm{Si}$ & & & $\mathrm{Si}$ \\
\hline S. glabratus & & & & Si \\
\hline S. jelskii & $\mathrm{Si}$ & & & $\mathrm{Si}$ \\
\hline S. latisquamus & $\mathrm{Si}$ & & & \\
\hline S. lundelli & $\mathrm{Si}$ & & & \\
\hline S. macroscyphus & $\mathrm{Si}$ & $\mathrm{Si}$ & & $\mathrm{Si}$ \\
\hline S. maculatus & $\mathrm{Si}$ & $\mathrm{Si}$ & & $\mathrm{Si}$ \\
\hline S. mcvaughii & $\mathrm{Si}$ & & $\mathrm{Si}$ & $\mathrm{Si}$ \\
\hline S. meridensis & $\mathrm{Si}$ & & $\mathrm{Si}$ & \\
\hline S. microcephalus & $\mathrm{Si}$ & & & $\mathrm{Si}$ \\
\hline S. oaxacanus & $\mathrm{Si}$ & & $\mathrm{Si}$ & \\
\hline S. obscurus & $\mathrm{Si}$ & & $\mathrm{Si}$ & \\
\hline S. parviceps & $\mathrm{Si}$ & & & $\mathrm{Si}$ \\
\hline S. putlanus & $\mathrm{Si}$ & & & $\mathrm{Si}$ \\
\hline S. pyramidalis & $\mathrm{Si}$ & $\mathrm{Si}$ & & $\mathrm{Si}$ \\
\hline S. quichensis & $\mathrm{Si}$ & & $\mathrm{Si}$ & \\
\hline S. riparius & $\mathrm{Si}$ & & & $\mathrm{Si}$ \\
\hline S. siegesbeckia & $\mathrm{Si}$ & & $\mathrm{Si}$ & $\mathrm{Si}$ \\
\hline S. sonchifolius & $\mathrm{Si}$ & $\mathrm{Si}$ & $\mathrm{Si}$ & $\mathrm{Si}$ \\
\hline S. uvedalius & $\mathrm{Si}$ & & & $\mathrm{Si}$ \\
\hline
\end{tabular}

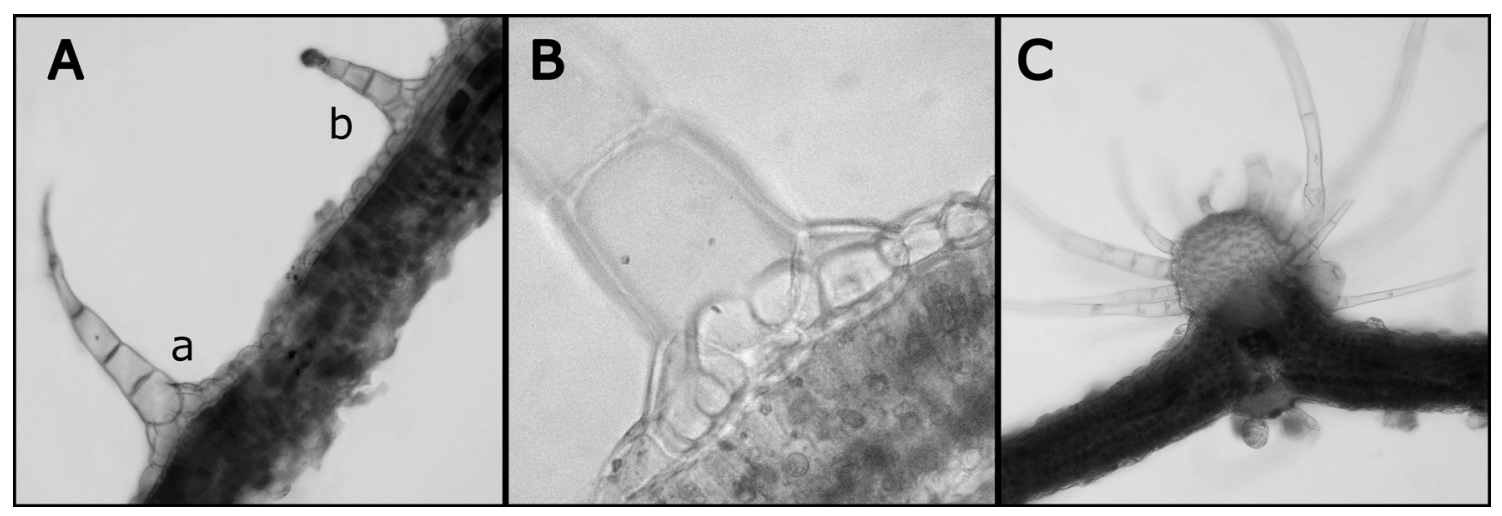

Fig. 5. Tipos de tricomas. A. Detalle de dos tipos de tricomas en S. obscurus (Breedlove 42387), tricoma eglandular a. y tricoma glandular b. B. Detalle del pie compuesto del tricoma eglandular del tipo cónico simple de S. meridensis (Steyermark 57519). C. Tricomas cónicos simples sobre protuberancias de las venas principales de las hojas en S. pyramidalis (King 10411). 


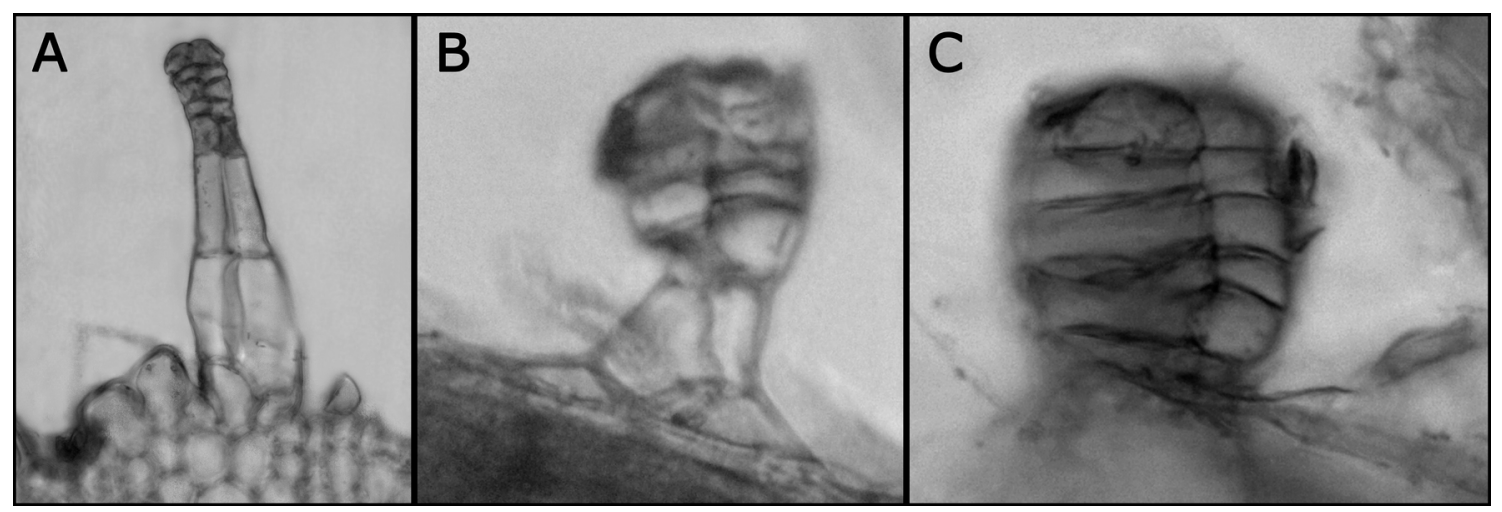

Fig. 6. Tricoma glandular biseriado vesiculado subtipo beta en Smallanthus. A. Tricoma glandular biseriado capitado en S. obscurus (Breedlove 42387). B. Tricoma biseriado vesiculado subtipo beta en S. connatus (Plos 46) donde se observa en detalle el pie del tricoma. C. Tricoma biseriado vesiculado subtipo beta en $S$. microcephalus (Sagástegui 14271) donde se observa en detalle el cuerpo.

en cada una de ellas. El análisis de la Tabla 3 muestra que existe una tendencia al aumento del número de canales, en aquellas especies que habitan las regiones más australes. Por ejemplo, $S$. connatus, que crece desde Brasil hasta las costas bonaerenses, posee en sus hojas de 6 a 10 canales, en tanto que $S$. uvedalius que se extiende desde el sudeste de los Estados Unidos hasta Panamá, posee un solo canal secretor. Hay excepciones, como es el caso de S. maculatus (Cav.) H. Rob., que se distribuye desde México hasta Panamá y posee hasta cinco canales secretores (Tabla 3). La tendencia al aumento del número de canales se cumple claramente en cuatro especies, S. connatus, S. macroscyphus, S. siegesbeckia y S. sonchifolius, que forman parte del denominado "Grupo yacón" de Grau y Rea (1997), ya que son las que poseen las distribuciones más australes del género y (Tabla 3) las que más canales secretores presentan. Sobre estas cuatro especies, se han llevado a cabo numerosas investigaciones, resultando todas con importantes propiedades medicinales y/o alimentarias (e.g. Aybar et al., 2001; Bach et al., 2007; Bresciani et al., 2004; Choque Delgado et al., 2013; Cabrera et al., 2007). Si bien, se podría decir que aquellas especies que poseen mayor número de canales secretores, poseerían propiedades medicinales y/o alimentarias, la Tabla 1 muestra otras especies del género que poseen usos medicinales de importancia, que no han sido estudiadas químicamente, y que según la Tabla 3 poseen bajos números de canales secretores. Una de ellas es la especie mexicana, S. mcvaughii (J. R. Wells) H. Rob., y que además presenta ambos tipos de pelos glandulares (Tabla 2) al igual que S. connatus, S. sonchifolius y $S$. siegesbeckia. Otra especie, con bajo número de canales, es Smallanthus pyramidalis (Triana) H. Rob., una de las dos especies arbóreas del género, que podría tener una importante función ecológica, ya que según Guzmán Avedaño \& Barrera Adame (2011), presenta metabolitos que funcionan como agentes atrayentes de polinizadores, entre otras funciones. Finalmente sería importante llevar a cabo estudios químicos también en otras especies, que se sabe poseen usos medicinales conocidos (Tabla1) tales como $S$. fruticosus (Benth.) H. Rob., S. meridensis (Steyerm.) H. Rob., S. parviceps (S. F. Blake) H. Rob. y S. maculatus. En el caso de S. quichensis (J. M. Coult.) H. Rob., que no tiene usos conocidos, se debería continuar investigando ya que sí se conoce la composición de los aceites esenciales de sus hojas (Chaverri \& Ciccio, 2015). Los resultados de este trabajo muestran que otras especies del género Smallanthus podrían ser igualmente o más valiosas que el "yacón", desde el punto de vista medicinal y/o alimenticio, por lo que sería importante llevar a cabo estudios que podrían corroborar o no las propiedades y usos de dichas especies. 
Tabla 3. Número de canales secretores

contabilizados de los cortes transversales de las venas de mayor calibre, de las hojas de todas las especies de Smallanthus y su distribución geográfica. * Por el estado del material en esta especie no se pudieron contabilizar los canales, pero sí se confirma la presencia de los mismos en el mesófilo.

\begin{tabular}{|c|c|c|}
\hline Especie & $\begin{array}{l}\text { Número } \\
\text { de } \\
\text { canales }\end{array}$ & Distribución \\
\hline S. connatus & $6-10$ & $\begin{array}{l}\text { Brasil, Paraguay, } \\
\text { Uruguay y Argentina }\end{array}$ \\
\hline S. cocuyensis & 1 & Colombia centro \\
\hline S. fruticosus & 3 & $\begin{array}{l}\text { Bolivia, Colombia, } \\
\text { Perú y Ecuador }\end{array}$ \\
\hline S. glabratus & $2-4$ & Bolivia y Perú \\
\hline S. jelskii & 4 & Perú \\
\hline S. latisquamus & 2 & $\begin{array}{c}\text { Costa Rica, Guatemala } \\
\text { y Mexico }\end{array}$ \\
\hline S. lundelli & 2 & Guatemala y Mexico \\
\hline S. macroscyphus & 4 & $\begin{array}{c}\text { Argentina, Paraguay, } \\
\text { Bolivia y Brasil }\end{array}$ \\
\hline S. maculatus & $2-5$ & $\begin{array}{l}\text { Desde Mexico } \\
\text { hasta Panamá }\end{array}$ \\
\hline S. mcvaughii & 2 & Mexico \\
\hline S. meridensis & $2-3$ & Venezuela \\
\hline S. microcephalus & * & Perú y Ecuador \\
\hline S. oaxacanus & $2-3$ & $\begin{array}{c}\text { Guatemala, Honduras } \\
\text { y Mexico }\end{array}$ \\
\hline S. obscurus & $2-3$ & Mexico \\
\hline S. parviceps & $2-3$ & Perú y Bolivia \\
\hline S. putlanus & 1 & Mexico \\
\hline S. pyramidalis & $2-3$ & $\begin{array}{l}\text { Venezuela, Colombia } \\
\text { centro y Ecuador }\end{array}$ \\
\hline S. quichensis & $1-2$ & Guatemala \\
\hline S. riparius & 3 & $\begin{array}{l}\text { Colombia centro, } \\
\text { Costa Rica, Ecuador, } \\
\text { Perú y Venezuela }\end{array}$ \\
\hline S. siegesbeckia & $4-6$ & $\begin{array}{c}\text { Ecuador, Perú, Bolivia, } \\
\text { Paraguay Brasil }\end{array}$ \\
\hline S. sonchifolius & 4 & $\begin{array}{c}\text { Bolivia y Perú (Argentina } \\
\text { sólo cultivado) }\end{array}$ \\
\hline S. uvedalius & 1 & $\begin{array}{l}\text { Estados Unidos } \\
\text { hasta Panamá. }\end{array}$ \\
\hline
\end{tabular}

\section{Agradecimientos}

Agradezco a los curadores de los herbarios que permitieron el estudio de los ejemplares como así también a los evaluadores anónimos. Mi especial mención es a la Dra. Liliana Katinas por su detallado análisis y acertadas sugerencias y recomendaciones que contribuyeron a mejorar este manuscrito. Este trabajo ha sido financiado por la Universidad Nacional de La Plata (UNLP), el Consejo Nacional de Investigaciones Científicas y Tecnológicas (CONICET) y La Agencia Nacional de Promoción Científica y Tecnológica ANPyCT.

\section{Bibliografia}

ASAMI, T., M. KUBOTA, K. M. MINAMISAWA \& T. TSUKIHASHI. 1989. Chemical composition of yacón, a new root crop from the Andean Highlands. Soil Sci. Plant Nutr. 60: 122-126.

AYBAR, M. J., A. N. SÁNCHEZ RIERA, A. GRAU \& S. S. SÁNCHEZ. 2001. Hypoglycemic effect of the water extract of Smallanthus sonchifolius (yacón) leaves in normal and diabetic rats. $J$. Ethnopharmacol. 74: 125-132.

BACH, S. M., C. SCHUFF, A. GRAU \& C. A. N. CATALÁN. 2007. Melampolides and other constituents from Smallanthus connatus. Biochem. Syst. Ecol. 35: 785-789.

BRESCIANI, L. F., R. A. YUNES, C. BÜRGER, L. E. DE OLIVEIRA, K. L. BÓF \& V. CECHINELFILHO. 2004. Seasonal variation of kaurenoic acid, a hypoglycemic diterpene present in Wedelia paludosa (Acmela brasiliensis) (Asteraceae). Z Naturforsch. 59:229-232.

CABRERA, W., A. DE PEDRO, M. E. PEROTTI, A. GRAU, C. CATALÁN, S. GENTA \& S. S. SÁNCHEZ. 2007. Hypoglycemic effect of Smallanthus macroscyphus leaves: identification of the active principle. Biocell 31: 311.

CHAVERRI, C. \& J. F. CICCIÓ. 2015. Composition of the essential oil from leaves of Smallanthus quichensis (Asteraceae) from Costa Rica. B. Latinoam. Caribe P. L. 14: 355-363.

CHOQUE DELGADO, G.T., W. M. C. TAMASHIRO, M. R. MARÓSTICA JUNIOR \& G. M. PASTORE. 2013. Yacón (Smallanthus sonchifolius): a functional food. Plant. Food. Hum. Nutr. 68: 222-228.

COLLARÁOZ, M.V., M. I. MERCADO, G. I. PONESSA \& A. GRAU. 2012. Morfología y anatomía foliar, caulinar y radicular de Smallanthus connatus (Heliatheae: Asteraceae). Lilloa 49: 22-29. 
COLL ARÁOZ, M.V., M. I. MERCADO, A. GRAU \& G. I. PONESSA. 2008. Morfología y anatomía foliar, caulinar y radicular de Smallanthus macroscyphus (Asteraceae). Lilloa 45: 23-33.

DE FORD, C., J. L. ULlOA, C. A. N. CATALÁN, A. GRAU, V. S. MARTINO, L. V. MUSCHIETTI \& I. MERFORT. 2015. The sesquiterpene lactone polymatin B from Smallanthus sonchifolius induces different cell death mechanisms in three cancer cell lines. Phytochemistry 117: 332-339.

GENTA, S. B., W. M. CABRERA, M. I. MERCADO, A. GRAU, C. A. CATALÁN \& S. S. SÁNCHEZ. 2010. Hypoglycemic activity of leaf organic extracts from Smallanthus sonchifolius: Constituents of the most active fractions. Chem. Biol. Interact.185: 143-152.

GRAU, A. \& J. REA. 1997. Yacón, Smallanthus sonchifolius (Poepp. \& Endl.) H. Robinson. In: HERMANN, M. \& J. HELLER (eds.), Andean roots and tubers: Ahipa, arracacha, maca and yacón, pp. 199-242. Institute of Plant Genetics and Crop Plant Research, Gatersleben/International Plant Genetic Resources Institute, Rome.

GUZMÁN AVENDAÑO, A. J. \& D. A. BARRERAADAME. 2011. Estudio fitoquímico de hojas y flores de Smallanthus pyramidalis (Triana) $\mathrm{H}$. Rob. (Arboloco) y su uso en la recuperación de los humedales de Bogotá. Colomb. For. 14: 41-50.

HOLMGREN, P. K., N. H. HOLMGREN \& L. C. BARNETT. 1990. Index Herbariorum, Part I: The herbaria of the world. 8th Edition, 693 pp. New York Botanical Garden, Bronx, New York.

INOUE, A., S. TAMOGAMI, H. KATO, Y. NAKAZATO, M. AKIYAMA, O. KODAMA, T. AKATSUKA \& Y. HASHIDOKO. 1995. Antifungal melampolides from leaf extracts of Smallanthus sonchifolius. Phytochemistry 39: 845-848.

LACHMAN, J., E. C. FERNÁNDEZ \& M. ORSÁK. 2003. Yacón [Smallanthus sonchifolia (Poepp. et End1.) H. Robinson] chemical composition and use a review. Plant Soil Environ. 49: 283-290.

MENDOZA MEZA, D. L. \& R. MEDINA VALDÉZ. 2015. Inhibición in vitro de las enzimas alfa-amilasa y lipasa pancreática por fracciones fenólicas de extractos etanólicos de hojas de Yacón (Smallanthus sonchifolius Poepp. \& Endl). Avances en Química 10: 33-40.
MERCADO, M. I., G. I. PONESSA \& A. GRAU. 2006. Morfología y anatomía foliar de "yacón", (Asteraceae), con fines de control de calidad. Acta Farm. Bonaerense. 25: 526-532.

MERCADO, M. I., M. V. COLL ARÁOZ, A. GRAU \& G. I. PONESSA. 2009. Morfología y anatomía foliar, caulinar y radicular de Smallanthus siegesbeckius (Heliantheae, Asteraceae). Lilloa 46: 77-87.

OHYAMA, T., O. S. YASUYOSHI, T. IKARASHI, K. MINAMISAWA, M. KUBOTA, T. TSUKINASHI \& T. ASAMI. 1990. Composition of storage carbohydrate in tuber roots of yacón (Polymnia sonchifolius). Soil Sci. Plant Nutr. 36: 167-171.

RAMAYYA, N. 1962. Studies on the trichomes of some Compositae I. General structure. Bull. Bot. Surv. India. 4: 177-188.

SEMINARIO, J., M. VALDERRAMA \& I. MANRIQUE. 2003. El yacón: fundamentos para el aprovechamiento de un recurso promisorio. Centro Internacional de la Papa (CIP), 60 pp. Universidad Nacional de Cajamarca. Agencia Suiza para el desarrollo y la Cooperación (COSUDE), Lima.

VALENCIA, G. \& M. ROMÁN. 2004. La fibra dietaria como alimento funcional. Vitae 11: 12-17.

VITALI, M. S. 2013. Revisión sistemática, análisis cladístico y biogeográfico del género Smallanthus Mack. (Asteraceae, Millerieae). Tesis de Doctorado. Facultad de Ciencias Naturales y Museo, Universidad Nacional de La Plata.

VITALI, M. S., G. SANCHO \& L. KATINAS. 2015. A revision of Smallanthus (Asteraceae, Millerieae), the “yacón” genus. Phytotaxa 214: 1-84.

Recibido el 14 de octubre de 2016, aceptado el 16 de diciembre de 2016. 


\section{ApÉNDICE}

ARGENTINA. Prov. Buenos Aires: Dpto. Magdalena, Magdalena, 08/12/2010, Plos et al., 46 (LP) S. connatus. Prov. Jujuy: Dpto. Capital, quebrada del Jaire, camino a Tiracsi, cultivado, 20/06/1962, Cabrera 14710 (LP) S. sonchifolius. Prov. Salta, Dept. Chicoana: Los Laureles, entrando a la Quebrada de Escoipe, frente a la planta de AGAS, 09/02/ 1987, Novara 5932 (S) S. macroscyphus. BOLIVIA. Dpto. La Paz: Prov. Larecaja, Sorata, 21/04/1920, Holway \& Holway 558 (GH) S. paviceps. BRASIL. Estado de Rio grande do Sul: São Francisco de Paula, Villa Oliva, 6/01/1946, Rambo 30992 (LP) S. connatus. COLOMBIA., Dpto. Cundinamarca, en cercanías de San Bernardo, hacia Sasaima, 23/06/1940, Cuatrecasas 9602 (F) S. fruticosus. Dpto. Caldas: Rio Quindio, 25/07/1922, Pennell 8731 (GH) S. riparius. Dpto. Cundinamarca: Valle del río Guavio, 4 km N de Ubalá, 29/05/1944, Grant \& Fosberg 9375 (F) S. cocuyensis. COSTA RICA. Dpto. Cartago: camino al Volcán Irazú, 23/08/1940, Chrysler 5544 (F) S. latisquamus. ECUADOR. Prov. Pichincha: carretera Quito-Nono-Tandayapa-Pto. Quito, en alrededores de Nono, $0^{\circ}$ 3' S, 78 $30^{\prime}$ W, 28/11/1987, Zak \& Jaramillo 3024 (K) S. fruticosus. ESTADOS UNIDOS. Estado de Kentucky: Condado de Harlan Southeastern, 8/1893 Kearney s/n (S) S. uvedalius. GUATEMALA. Dpto. Baja Verapaz: Unión Barrios, 27/02/ 1972, Contreras 11065 (S) S. lundelli. Dpto. Huehuetenango: Ixtatán, cerca de Kurus Lemun, 4 miles E of San Mateo Ixtatán, en el camino a Barillas, 07/08/1965, Breedlove 11598 (F) S. quichensis; Dpto. San Marcos: Barranco Eminencia, camino entre San Marcos y San Rafael Pie de la Cuesta, 06/02/1941, Standley 86364 (F) S. quichensis. HONDURAS. Dpto. Ocotepeque: Cordillera Merendón, 10 km de Nueva Ocotepeque, camino a El Portillo, 26/08/1968, Molina 2225 (F) S. oaxacanus; Dpto. Morazán La Montañita, 18/11/1948, Standley 14781 (F) S. oaxacanus. MEXICO. Estado de Oaxaca: Mpio. de Santa Cruz Itundujia,a 0.5 km en LR (W) de la Agencia de la Victoria, ca.1091 m, 16 44' 7' N, 97 45'36" W, 2/08/2008, Gutiérrez et al., 3099 (Holótipo de S. putlanus TEX). Mpio. de San Cristobal, Las Casas, along road to Chanal, 16-20 Km E of Chilil, 2380 m, 10/11/1976, Breedlove 42387 (Holótipo de $S$. obscurus TEX). Estado de Mexico, Mpio. de Naucalpan: 4 km al E de Chimalpa, carretera Mexico-Toluca, Naucalpan. 03/09/1967, Hidalgo s/n. (LP) S. maculatus; Estado de Veracruz: Mpio. de Huatusco: Santiago Tuxtla, cerro Blanco al NW de Santiago Tuxtla, 09/08/1972, Beaman 6425 (F) S. maculatus. Estado de Jalisco: Estación de Biología Las Joyas, en la Sierra de Manantlán, entrando por El Chante, carr. Autlan-El Grullo, 19 34' N, 104 14' W, 09/03/1992, Campos 4563 (GH) S. mcvaughii. NICARAGUA. Dpto. Zelaya. Área del Ocotal, río Grande, 23/04/ 1949, Molina 2338 (F) S. maculatus. PARAGUAY., Dept. Misiones: Santiago, Estancia La Soledad, 13/12/ 1969, Pedersen 9551 (S) S. connatus. PERÚ. Prov. Junín. Dpto. Junín: Chanchamayo, 15/10/1863, Isern 2272 (F); Tarma 01/03/1948, Woytkowski 35402 S. siegesbeckia (F). Prov. Cajamarca, Dpto. Huánuco: Huánuco, 1824, Ruiz et Pavon s/n. (F) S. fruticosus; Prov. Chota Chota-Bambamarca, 27/051965, López \& Sagástegui 5471 (LP) S. jelskii; Dpto. Lima: Chosica, 28/04/ 1922, Macbride \& Featherstone 1554 (F) S. glabratus. Prov. Celendín, Guañambra-Sendamal, 19/08/1984, Sagástegui 12197 (F) S. jelskii. Prov. Contumazá, El Molino (Cascas-Contumazá), 31/06/1990, Sagástegui 14271 (TEX) S. microcephalus. VENEZUELA. Estado de Mérida: Mpio. de Miranda, Chachopo, 2045 m, 22 July 1944, Steyermark 57519 (Holótipo de S. meridensis F); Mpio. de Rangel a 12 km al E de Tabay, 17/01/1993, King 10411 S. pyramidalis $(\mathrm{GH})$. 